\title{
Intelligent massage chair based on blood pressure and heart rate
}

\begin{abstract}
Massage is one of the effective techniques used to relieve pain, reduce stress, and increase relaxation. Nowadays, massage methods have varied to an easier way by electronic massage chair massage which is able to control the massage mode according to specifications. Commercial massage chair can only be used on normal healthy people with no health complication, such as diabetes, heart problem or blood pressure problem. The purpose of this study is to develop a system of more advanced massage chair where it can have an impact on user health based on blood pressure and heart rate. The proposed massage chair equipped with blood pressure and heart rate sensors, where the therapy will automatically configured according to individual's blood pressure and heart rate readings. The proposed control system developed using the artificial intelligent of fuzzy logic for data classification. The complete model of this massage control system is then developed using SIMULINK software to simulate the whole system operation and verify the function as programmed. Upon the success of the investigation, the findings will be useful for rapid advances in the existing technology.
\end{abstract}

Keyword: Blood pressure; Deep tissue massage; Fuzzy logic; Heart rate; Massage chair 